\title{
On 3D Scanning, Reconstruction, Enhancement, and Segmentation of Logs
}

\author{
Katarina Flood, Per-Erik Danielsson, and Maria Magnusson Seger \\ Computer Vision Laboratory, Linköping University, Linköping, Sweden \\ \{katarina, ped, maria\}@isy.liu.se
}

\begin{abstract}
This paper presents novel results from an ongoing feasibility study of fully 3D X-ray scanning of Pinus Sylvestris (Scots Pine) logs. Logs are assumed to be translated through tw o iden tical and static cone beam systems with the beams rotated $90^{\circ}$ relative to eachother, providing a dual set of 2D-projections. For reasons of both cost and speed, each 2D-detector in these tw o systems consists of a limited number of line detectors. The quality of the reconstructed images is far from perfect, due to sparse detector data and missing projection angles. In spite of this we show that by employing a shape- and direction discriminative technique based on second derivativ es, w e are able to enhance knot-lik features in these data. In the enhanced images it is then possible to detect and localize the pith for each whorl of knots, and subsequently also to perform a full segmentation of the knots in the heartwood.
\end{abstract}

\section{Introduction and Overview}

F or sawmills, a key observation is that the qualit yand price of a board to a large extent depend on the size and frequency of defects, mainly knots. Given the locations, sizes, and shapes of the knots, it w ould be possible to optimize the sa wing pattern for maximum gain. Such a procedure has been estimated [1] to increase the sales value of sawn boards considerably, possibly up to $10 \%$.

$\mathrm{X}$-ray computed tomography $(\mathrm{CT})$ is a well established field in medical imaging. Since wood has a density variation similar to human tissue because of the abundance of w aterand organic material, CT should be equally suitable for imaging of logs. Conven tional medical tomographs are designed for highest possible image quality and has a maximum translational speed of less than $10 \mathrm{~cm} / \mathrm{s}$. In a sawmill, however, logs are often translated (length-wise) at 2-3 m/s, which indicates that a completely different method for acquiring projection data is necessary. As will be shown in the next section, the data captur edevice we envision con tains no rotating gantry but tw o fixed cone-beam systems collecting relatiely sparce data. This scanning geometry requires an unconven tionalreconstruction algorithm developed for this very specific purpose. Let us emphasize that the scanning and data capture systems have not been implemented ph ysically. All results stem from simulated projection data obtained by computer simulated Xray exposure of a digitized log volume reconstructed from a high quality medical tomograph. 
Clearly, the relatively cheap data capture system will sacrifice image quality for speed. As an example, due to the low number of projections, the images will contain so called streak artifacts, which turn the segmentation problem into a real challange. Fortunately, we found it possible to enhance the reconstructed images, ridding them from most streak artifacts and at the same time retaining and enhancing the knot features. Second derivatives form the basic tool in this step of the total procedure.

The next step is to find the pith, the (approximate) center of the growing tree from which all branches once originated as sprouts, bringing about a new whorl every year. Therefore, each knot is "rooted" in the pith and that is where the segmentation of each knot should start. The robust pith finding algorith presented below utilizes the orientation information embedded in the second derivatives. Given the pith position for a whorl, and the relatively good objectto-background contrast, it is not too difficult to make a unique labeling and final segmentation of the knots in the heartwood of the log.

Unfortunately, the contrast in both the original and the enhanced data drops dramatically in the sapwood. Therefore, the successfully segmented and labeled knot cannot easily be processed outwards from the heartwood into the sapwood. At the time of this writing, we are testing various methods to solve this remaining step in the full-fledged segmentation of knots in Pinus Sylvestris.

\section{New Scanning Geometry and Corresponding Reconstruction Algorithm}

The scanning geometry can be seen as an extension of the LogScanner, described in [2]. It was first described in [3] and is illustrated in Fig. 1 together with the corresponding reconstruction algorithm. The log is translated with high speed (2$3 \mathrm{~m} / \mathrm{s}$ ) through an arrangement of two X-ray sources and two area detectors, each of them consisting of a number of sparcely placed line detectors. The projection data from each source-detector system is first reconstructed individually and then merged into one reconstruction volume, showing the interior of the log.

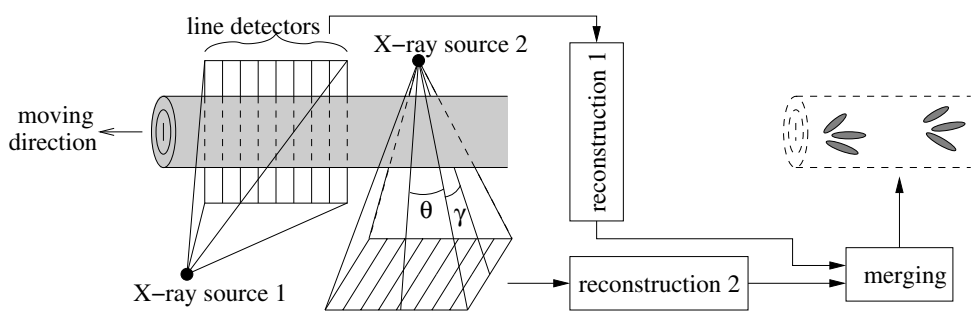

Fig. 1. Illustration of linear cone-beam tomography for logs. 
The reconstruction algorithm starts with rebinning as shown in Fig. 2a). The original cone-beam projection data is then resorted into fan-parallel data, which is fan-shaped in one direction and parallel in the other. In the figure, we see how one element in detector no 2 (and 3) collects parallel line integrals while the log is translated in the horizontal direction. The projections are oriented in a manner of the sheets of an half-open book. The rays 1, 2, 3, 4, 5 in Fig. 2a) are all in one plane (or sheet) and therefore we are allowed to employ $2 \mathrm{D}$ reconstruction for each such plane at a time. The $2 \mathrm{D}$ reconstruction can be done by a Fourier method as described in [3] or by filtered backprojection, see for example [5]. Putting the reconstructed sheets together gives one reconstructed volume.

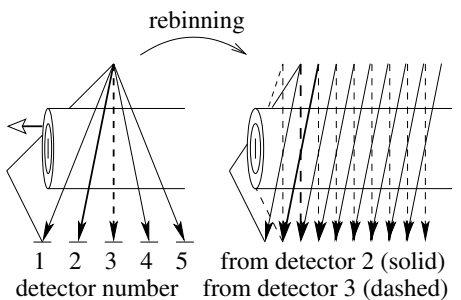

a)

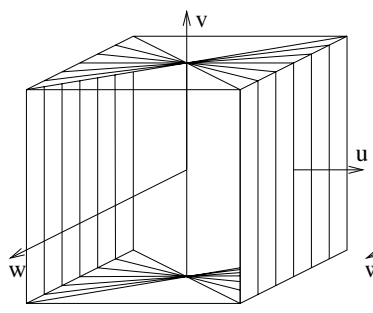

b)

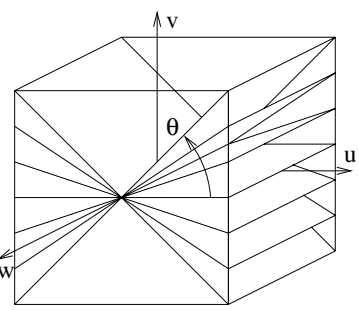

c)

Fig. 2. a) Rebinning. b), c) Projection data in the 3D Fourier domain from sourcedetector system 1 (b) and 2 (c).

Merging of the two reconstruction volumes is not trivial. Assume for a moment that the projections are fully parallel. According to the well-known Fourier slice theorem, the 2D Fourier transform of a parallel projection through the object is equal to the 3D Fourier components of the object found in a plane through the origin perpendicular to the projection rays. See Fig. 2b) and c) showing projection data in the 3D Fourier domain from source-detector system 1 and 2, respectively. The number of detectors is here assumed to be $2 \times 7$ and the angular interval is $-45^{\circ} \leq \theta \leq 45^{\circ}$. Some parts of the Fourier domain are covered by one system, some parts are not coved by any system (missing data), and some parts are covered by both. The data in the overlapping regions should be weighted with a factor of 0.5 . Merging the reconstructed fan-parallel data instead of true parallel data in the $3 \mathrm{D}$ Fourier domain will be more or less approximate depending or the fan-angle $\gamma$.

Fig. 3 b) and d) show reconstruction results with $2 \times 9$ line detectors and Fig. 3a) and c) show the corresponding normal CT-images for comparison. While the knots are clearly visible, the heart- and sapwood have disappeared, because of the missing data in the 3D Fourier domain. The image quality is better in the heartwood than in the sapwood since the contrast between knots and surrounding wood is higher there. Note also the streak artifacts caused by the low number of detectors. Due to the system geometry, only features with variation in the length-wise direction of the log can be captured. 


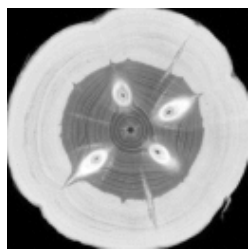

a,

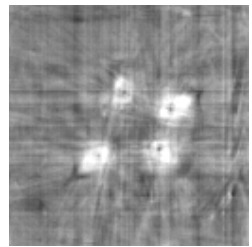

b,

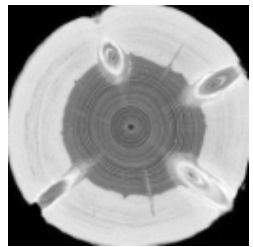

C,

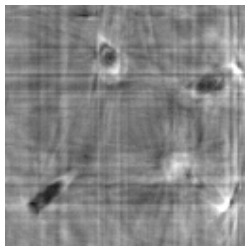

d,

Fig. 3. Reconstruction results for two different slices. a), c) CT-images for comparison. b), d) Reconstruction results for $2 \times 9$ line detectors.

\section{Enhancement and Shape Discrimination using Second Derivatives}

For the necessary enhancement step we have chosen a low-level technique based on second derivatives, which allows for both shape and orientation discrimination. The six second degree derivatives are computed by convolving the input volume $f(x, y, z)$ with the six derivative operators $g_{x x}, g_{y y}, g_{z z}, g_{x y}, g_{x z}, g_{y z}$ and then assembling the result in the Hessian $H$.

$$
H=\left(\begin{array}{lll}
f_{x x} & f_{x y} & f_{z z} \\
f_{x y} & f_{y y} & f_{y z} \\
f_{x z} & f_{y z} & f_{z z}
\end{array}\right)
$$

Several authors have extracted shape information from the Hessian eigenvalues after sorting them according to their magnitudes, as proposed by [6]. However, since the Hessian is indefinite, we sort then as they appear with signs [4]. The three eigenvalues $\lambda_{1}>\lambda_{2}>\lambda_{3}$ then correspond to the second derivatives $p_{x x}, p_{z z}, p_{y y}$ of a rotated version $p(x, y, z)$ of the original neighborhood $f(x, y, z)$ with all threee cross-derivatives equal to zero. The second derivative operators do not form an orthonormal basis [4], and thus we orthonormalize their responses (the three eigenvalues) in Eq.2. Note that we have chosen a very specific order that relates each eigenvalue to the derivatives of the derotated neighborhood, and thereby also to the coordinate system of the signal space.

$$
\mathbf{p}=\left(\begin{array}{l}
p_{20} \\
p_{21} \\
p_{22}
\end{array}\right)=\left(\begin{array}{ccc}
\frac{1}{\sqrt{6}} & \frac{1}{\sqrt{6}} & \frac{1}{\sqrt{6}} \\
-\sqrt{\frac{5}{24}} & \sqrt{\frac{5}{6}} & -\sqrt{\frac{5}{24}} \\
\sqrt{\frac{5}{8}} & 0 & -\sqrt{\frac{5}{8}}
\end{array}\right)\left(\begin{array}{c}
\lambda_{1}=p_{x x} \\
\lambda_{2}=p_{z z} \\
\lambda_{3}=p_{y y}
\end{array}\right)
$$

Thanks to the orthonormalization, the derotated second derivative response $p$, deliberated from all orientation influence carried along in $f$, is now a vector that can be studied in the shape space shown in Fig. 4. This 3D-space is spanned by the three basis functions $c_{20}, c_{21}, c_{22}$, which are related to the three derivative operators $g_{x x}, g_{y y}, g_{z z}$ in the same manner as the three components $p_{20}, p_{21}, p_{22}$ in Eq.2 are related to $p_{x x}, p_{y y}, p_{z z}$.

All shapes are located on a $60^{\circ}$ sector of the unit sphere. The magnitude of $\mathbf{p}$ is the length of the vector, while the longitudinal angle $\frac{\pi}{3} \leq \eta<\frac{2 \pi}{3}$ and the latitude angle $\frac{-\pi}{2} \leq \kappa<\frac{\pi}{2}$ defines the shape. The position of some archetypical 3D-shapes are indicated in Fig.4, namely blobs, strings, and planes of both polarities. 


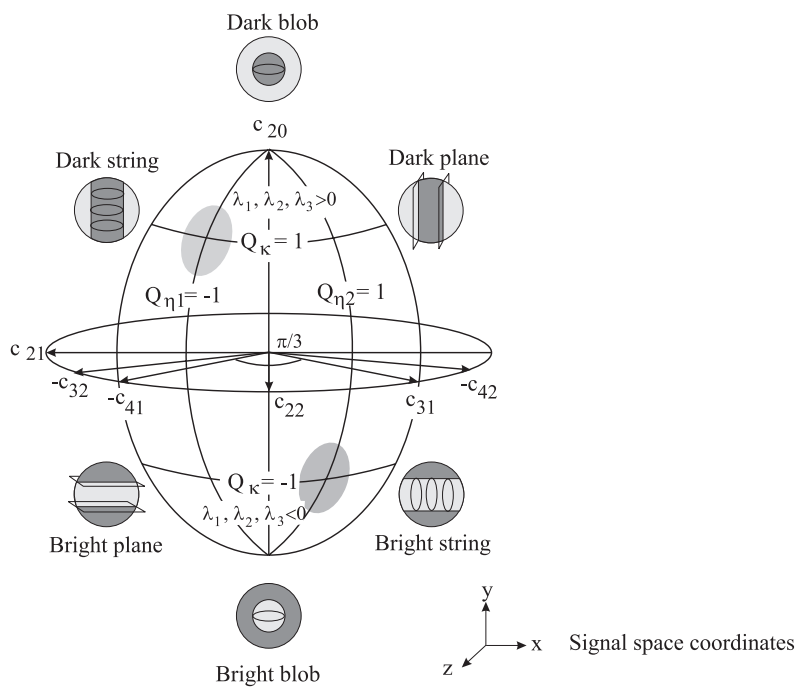

Fig. 4. An outline of the second degree variation shape space. The two gray spots on the surface mark where the shape discriminaing factor $Q_{\kappa \eta}(x, y, z)$ takes on values close to \pm 1 , i.e. for bright and dark strings, respectively.

It is possible to discriminate for different shapes in this space. If, for example, the vector $\mathbf{p}$ points toward the shape "bright string", the local second degree variation of $f(x, y, z)$ in the neighbourhood of $(x, y, z)$ is indeed a bright string, rotated according to the eigenvector matrix in Eq.2.

A knot is basically string-shaped although its cross-section is sometimes elliptic. Therefore, for voxels belonging to a typical knot, we expect $\mathbf{p}$ to point toward either of the two highlighted regions in Fig. 4. To identify these two regions we first compute four linear combinations of the available responses (Eq.3).

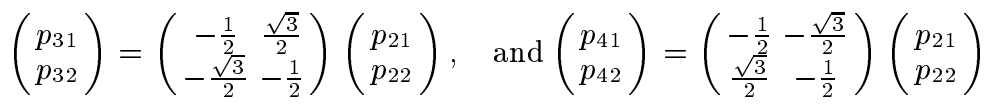

We can then define a shape discriminating factor $Q_{\kappa \eta}$ [7], which takes on maximum/minimum values \pm 1 for bright and dark strings respectively, and tapers off at a suitable pace outside the two regions.

$$
\begin{gathered}
Q_{\kappa}=\frac{2 p_{20} \sqrt{p_{21}^{2}+p_{22}^{2}}}{p_{20}^{2}+p_{21}^{2}+p_{22}^{2}} \\
Q_{\eta 1}=\frac{2 p_{31} p_{32}}{p_{31}^{2}+p_{32}^{2}}, Q_{\eta 2}=\frac{2 p_{41} p_{42}}{p_{41}^{2}+p_{42}^{2}} \\
Q_{\kappa \eta}= \begin{cases}Q_{\kappa} Q_{\eta 1} & \text { if } p_{20}>0 \\
-Q_{\kappa} Q_{\eta 2} \text { if } p_{20} \leq 0\end{cases}
\end{gathered}
$$

Because of reconstruction artifacts and the wish to only detect strings of high magnitude, we compute the final string response $s(x, y, z)$ as

$$
s(x, y, z)=\left\|\mathbf{p}_{2}(x, y, z)\right\| Q_{\kappa \eta}(x, y, z) .
$$


Two examples of such string enhancement on the log data in Fig. 3 are shown in Fig 5.

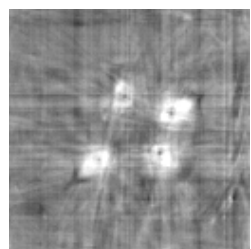

a,

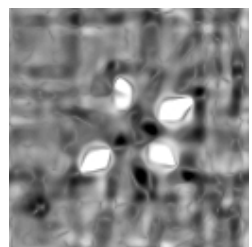

b,

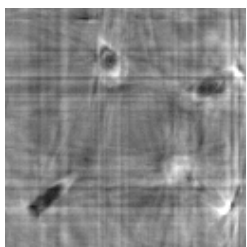

C,

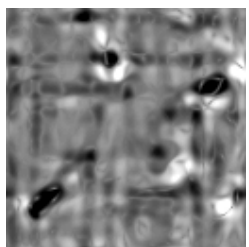

d,

Fig. 5. String enhancement results for two different slices. a), c) Reconstructed images using $2 \times 9$ line detectors for comparison (see Fig.3). b), d) String enhancement results.

The direction of the string is taken as the eigenvector $\mathbf{u}=\left(u_{x}, u_{y}, u_{z}\right)$ of the smallest eigenvalue.

Naturally, knots are of varying size, depending on location and also individual properties of the log. Therefore, we detect knots in a scale hierarchy, i.e. use derivative operators of different sizes to estimate the Hessian. These results are then combined.

\section{Pith Finding and Subsequent Segmentation of Knots}

The knots in Pinus Sylvestris are arranged in whorls. All knots in one whorl originate from the pith, and the detection of its location is a major initial step in segmentation of the knots. However, the reconstructed images are of low quality and quite often it is not possible to find the pith even manually.

A one dimensional intensity profile $I(z)$ of the $\log$ (see Fig. 6) can reveal where knot whorls are located along the lengthwise $(z-)$ direction. Such a profile can easily be obtained from the original rebinned projections.

There are well-defined intervals $\Delta z_{i}$ in $I(z)$ of high intensity, corresponding to the knot whorls. Each volume defined by one of these intervals is subjected to the string enhancement procedure described above, which delivers magnitude $s(x, y, z)$, and direction $\mathbf{u}(x, y, z)$.

Ideally, all lines, each one drawn from a string voxel $(x, y, z)$ in the string direction $\mathbf{u}(x, y, z)$ intersect at one point, the actual pith. In reality, this is not true. A least square error search is needed to find the point that is most likely to be the pith. This is done as follows.

We threshold the string enhanced image $s(x, y, z)$ with a threshold $\mathrm{T}$ to only capture very strong strings, and perform a summation over $z$ as in Eq. 8 .

$$
\mathbf{u}_{2}(x, y)=\left(\begin{array}{l}
u_{x} \\
u_{y}
\end{array}\right)(x, y)=\int_{z}(s(x, y, z)>T)(s \mathbf{u})(x, y, z) d z
$$

The equation of the line (see Fig. 7$) l(x, y)$ passing through pixel $(x, y)$ is

$$
l(x, y)=x \cos \varphi+y \sin \varphi
$$

where $\varphi$ is the angle between the positive $x$-axis and the normal to the line. 


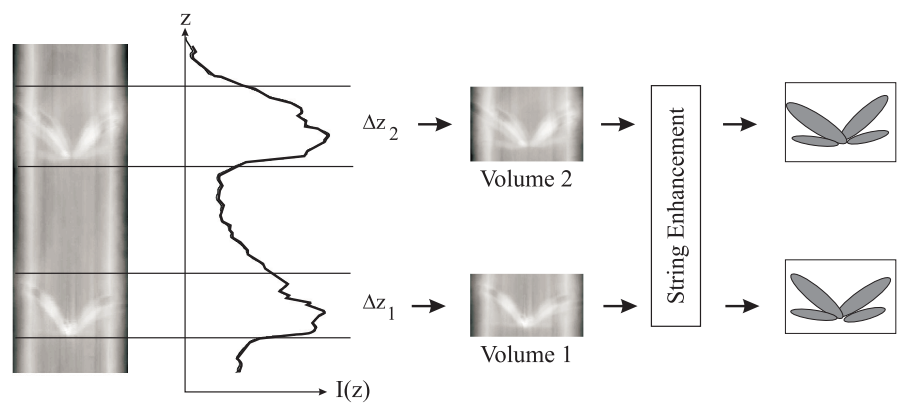

Fig. 6. An intensity plot $I(z)$ makes it possible to locate regions $\Delta z_{i}$ where whorls are located. The $3 \mathrm{D}$ volumes defined by the $\Delta z_{i}$ are then subjected to string enhancement.

The $z$-coordinate of the pith can be found in an intensity profile of $s(x, y, z)$. Thus remains to determine the coordinates $\left(x_{0}, y_{0}\right)$, which minimizes the function

$$
g\left(x_{0}, y_{0}\right)=\sum_{i}\left(l_{i}-x_{0} \cos \varphi_{i}-y_{0} \sin \varphi_{i}\right)^{2} .
$$

This is a least square problem which can be solved explicitly by setting the partial derivatives $g_{x_{0}}$ and $g_{y_{0}}$ to zero.

$$
\begin{aligned}
& g_{x_{0}}=2 \sum_{i} x_{0} \cos \varphi_{i}{ }^{2}+y_{0} \sin \varphi_{i} \cos \varphi_{i}-l_{i} \cos \varphi_{i}=0 \\
& g_{y_{0}}=2 \sum_{i} y_{0} \sin \varphi_{i}{ }^{2}+x_{0} \sin \varphi_{i} \cos \varphi_{i}-l_{i} \sin \varphi_{i}=0
\end{aligned}
$$

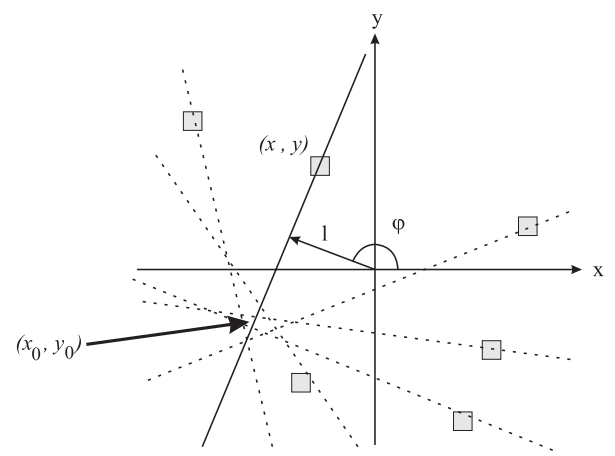

Fig. 7. The lines defined in each pixel in $\mathbf{u}_{2}(x, y)$. An individual pixel $(x, y)$ defines a line passing through it with slope $\arctan \frac{u_{y}}{u_{x}}$, and angle $\varphi$ of the normal to the line. The pith $\left(x_{0}, y_{0}\right)$ is the point which minimizes Eq. 10.

The procedure was applied to a whorl volume, slices of which were shown in Figs. 3 and 5 with a result shown in Fig. 8a).

Labeling of individual knots is the next goal. We produce a scalar binarized version $b(x, y)$ of the vector image $\mathbf{u}_{2}(x, y)$ by thresholding its amplitude. A radial projection of $b(x, y)$, or rather the number of non-zero pixels in $b(x, y)$ are 


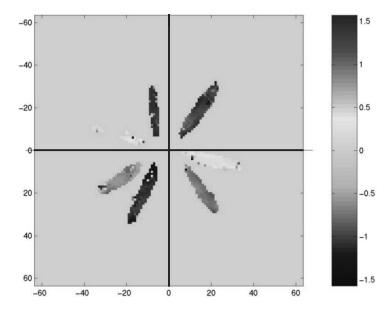

a,

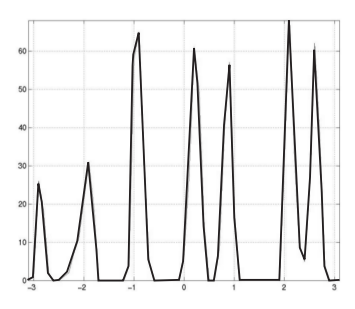

b,

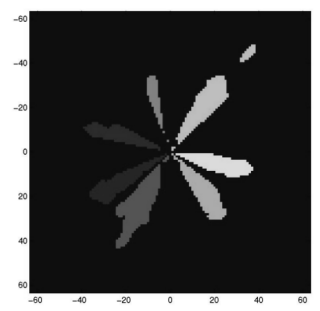

$\mathrm{c}$,

Fig. 8. The pith finding algorithm and succeeding segmentation of the knots in the heartwood. (a) shows the angular value of $\mathbf{u}_{2}(x, y)$. When the pith is found, a radial projection $P_{r}$ is calculated (b), and segmentation of the knots is possible (c).

then calculated for a discrete number $N$ of angular intervals $\psi_{i}$. See Fig. 8b).

$$
\begin{gathered}
P_{r}\left(\psi_{i}\right)=\int_{0}^{R} b\left(r, \psi_{i}\right) d r, \text { where } \\
r=\sqrt{x^{2}+y^{2}}, \\
\psi=\arg (x, y)
\end{gathered}
$$

Each peak in $P_{r}$ should correspond to a knot, and we have found that this is true even for low quality volume data. By thresholding $P_{r}$ with its mean, we obtain a unique label for each knot. These labels are then back-projected into all non-zero pixels in $b(x, y)$, see Fig.8c). This segmentation is subsequently transferred also to $3 \mathrm{D}$, and concludes the segmentation of the knots in the heartwood.

\section{Conclusions}

We have described a geometry and subsequent reconstruction algorithm designed for 3D X-ray scanning of logs in a sawmill process. In was shown that it is possible to detect the pith of each whorl in the reconstructed images using a shape and direction sensitive technique based on second derivatives. Segmentation of the knots in the heartwood was performed using a radial projection of the whorl.

\section{References}

1. L.G. Johansson and Å. Liljeblad, Some applications withing the project "Quality simulation of sawn logs", Trätek, Sweden, 1988, I 880605

2. Stig Grundberg. An X-ray log-scanner - a tool for control of the sawmill process. PhD-thesis, Luleå University, Sweden, 1999.

3. Maria Magnusson-Seger, Per-Erik Danielsson. Linear Cone-Beam Tomography for Logs, In the 4th International Conference on Image Processing and Scanning of Wood, 2000.

4. Per-Erik Danielsson, Qingfen Lin, Qin-Zhong Ye. "Efficient detection of second degree variations in 2D and 3D images". Journal of Visual Computations and Visual Representation, 2001.

5. A. C. Kak and M. Slaney Principles of Computerized Tomographic Imaging IEEE Press, New York, 1988.

6. T. Koller From Data to Information: Segmentation, Description, and Analysis of the Cerebral Vascularity PhD-thesis, Swiss Federal Inst. of Techn., Zurich, 1994.

7. K.Flood and P.E. Danielsson Detection of knots in 3D log scanning, Proc. of 2001 Symposium in Image Analysis, 2001. 